\title{
Monthly Weather Forecasting through ANN Model: A Case Study in Barisal, Bangladesh
}

\author{
Taohidul Islam ${ }^{1}$, Sajal Saha ${ }^{2}$, Ali Ahmed Evan ${ }^{3}$, Nabonita Halder ${ }^{3}$, Shakti Chandra Dey ${ }^{3}$ \\ Associate Professor, Dept of Electrical and Electronics Engineering' Patuakhali Science and Technology University ${ }^{1}$ \\ Lecturer, Department of Computer and Communication Engineering, Patuakhali Science and Technology University ${ }^{2}$ \\ Research Students, Faculty of Computer Science and Engineering, Patuakhali Science and Technology University ${ }^{3}$
}

\begin{abstract}
Bangladesh demonstrates seasonal alterations with six seasons where natural calamities cause tragic death of lives and severe hazardous in Bangladesh regularly and frequently. It is difficult to predict the weather pattern because of non-linear characteristics of natural disaster and seasonal changes in the country. The conventional weather prediction models are conducting in a huge domain and high resolution in Bangladesh which is constraint to show reliable seasonal disaster predictions. The research uses a methodology that considers specific and significant weather parameters from meteorological data in MATLAB platform to train the ANN based model. This paper includes a case study on prediction of heavy rainfall in Barisal City of Bangladesh using the model of artificial neural network (ANN) for monthly basis reliable weather forecasting planning. The model has been tested with individual month's average rainfall percentage to obtain the confirmation of the model. The results based on mean square error function (MSE) confirm the reliability of model based on multilayer perceptions for weather forecasting with successful application. The results are compared with weather information of FORECA limited to validate the seasonal disaster prediction criteria. This paper is important for the preparedness of people about forthcoming disaster in Bangladesh.
\end{abstract}

Keywords: Pre disaster prediction, ANN model, weather parameters, seasonal variations.

\section{INTRODUCTION}

Natural disasters like tropical cyclone, storm surges, flood, heavy rain, tornadoes cause a direct impact on the safety and economic stability of Bangladesh. The entire economic of the country is based on agricultural product and agriculture depends upon weather [4-5,10]. The main purpose of weather forecasting is to alert people about upcoming disaster so that proper steps can be taken to protect lives and properties. Disaster prediction in Bangladesh is constraint for due to the lack of proper selections of imprecise weather parameters, quicker seasonal variations in Bangladesh [2,7]. Rahman and Matin [18] shows the comparison of ANN model and other statistical model for monsoon rainfall prediction where ANN model is found as superior than other statistical model.

Mahapatra [13] has used ANN for weather prediction including flood prediction. ANN is able to automatically map the relationship between the stored parameters [6]. Rahman and Haque [14] shows fuzzy system based on weather prediction for Bangladesh. They considered wind speed for measuring cyclone danger level where wind speed range is divided into 9 categories. It includes fuzzy algorithms to get the fuzzy output to determine the best range of wind speed where best range is converted to a percentage of the danger level. Using Fuzzy based logic with the categories of numerical data overlapping shows $84 \%$ of accuracy in weather prediction [14].

Another research by Janal, et al. [8] on operative flash floods prediction used artificial intelligent methods with a hydrological model which is able to estimate the resultant peak outflow. Fuzzy interface system algorithm was used for the modeling where the problems of flash floods can be solved using artificial intelligence methods. A survey on rainfall prediction using ANN shows that Back Propagation Network, Radial Basis Function Networks, Super Vector Machine and Self Organizing Map are the most commonly for several forecasting technique [13]. In another literature discussed on several types of flood forecasting systems used Artificial Neural Network and Support Vector Machine in the model $[3,11]$. Suliman, et al.[16] shows that ANN are well suited for problems which have enough data or observations to see the trend for predictions. In another paper by Agrawal, et al.[1] revealed that ANN has the important features of adaptively, robustness, power and non-linearity to analyze satellite data for cyclone tracking.

Roy [15] proposed cyclone forecasting model based on satellite images using ANN for satellite images processing. A multi-layered neural network was trained to forecast the movement direction of cyclone where cloud image was processed and categorized using ANN.

Kumar and Jha [12] discussed on a time series ANN approach for weather forecasting with maximum and minimum temperature. Kadu, et al. [9] proposed a model of temperature prediction through wireless technology interfacing with statistical software for data collection. Intelligent Weather Monitoring System was developed by Imran, et al. [6] based on the parameters of one year's daily temperature and wind speed. Armesh and Negaresh 
[17] used ANN based simulation for daily rainfall prediction of Saravan-Iran. Though ANN based weather prediction model is shown by other research group, most of the research include huge abundant data and extensively study to obtain the meteorological simulation and forecasting. Therefore, it is difficult to get specific and reliable seasonal weather forecasting through the conventional criteria of disaster prediction.

Feed-forward MLP networks are used in our developed model with input, hidden and output layers. This network is included to obtain a model with reliable outcomes using historical data. The model is important to produce the output when the desired output is unknown. MLPs are the most common type of feed-forward networks. Neurons in input layer act as buffers for distributing the input signals $x_{i}(i=1,2 \ldots . . n)$ to neurons in the hidden layer for MLPs. Each neuron $\mathrm{j}$ in the hidden layer sums up its input signals $\mathrm{x}_{\mathrm{i}}$ after weighting them with the parameters $\mathrm{w}_{\mathrm{ji}}$ from the input layer and its output $\mathrm{y}_{\mathrm{j}}$ is taken as a function $f$ of the sum.

$$
y_{j}=f\left(\sum_{i=1}^{n} w_{j i} x_{i}\right)
$$

Where, $\mathrm{f}$ can be a simple threshold function or a sigmoidal, hyperbolic tangent or radial basis function. The output of neurons in the output layer is computed similarly.

\section{METHODOLOGY}

Most of the part of Barisal city of Bangladesh was flooded for heavy rainfall from June 062015 to June 10, 2015. Roads, organizations and offices were blocked by rain water as seen in Figure 1. Sudden flood causes great loss for some middle class businessmen. The investigation shows that the lack of flood prediction and proper drainage system of Barisal city cause such sudden water congestion in Barisal city especially in the central part of the city as shown in Figure 1.

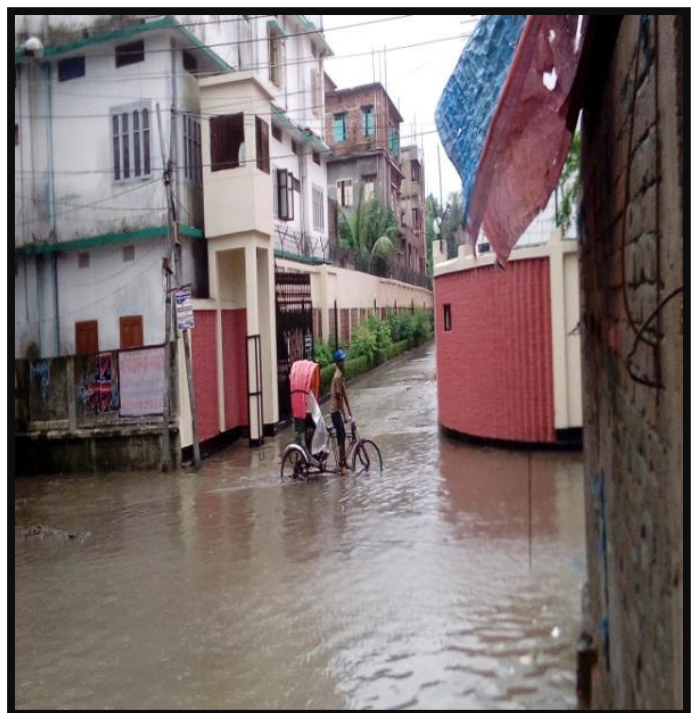

(a)

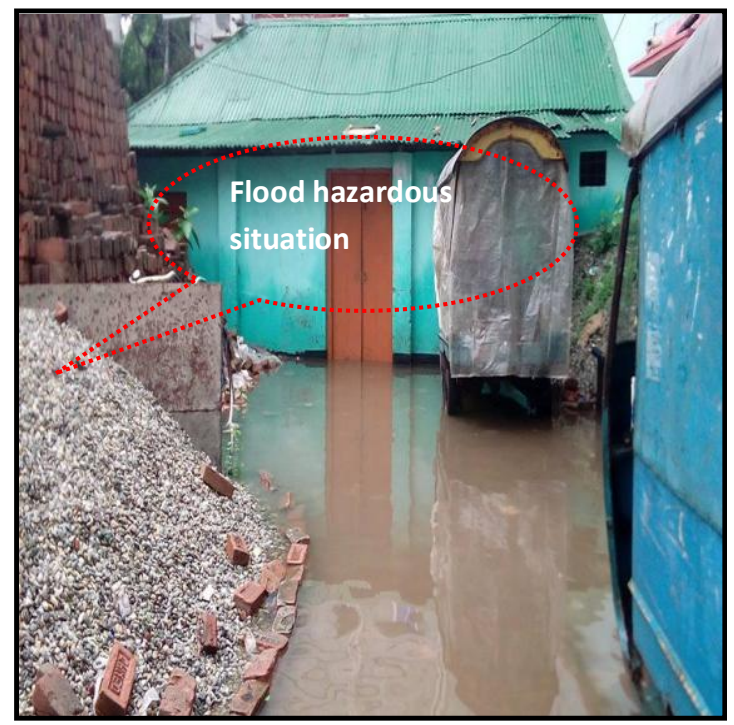

(b)

Figure 1 Flooded for heavy rainfall in Barisal City (a) front of Bangladesh bank (b) Metro city of Barisal

Weather data is obtained from Gopalgang station and verified from Barisal weather office. Weather data of month April to November, 2015 are used as input and target data for programming based analysis. To carry out the case study in Barisal city, input parameters are given in the network as (a) daily minimum and maximum temperature $\left({ }^{\circ} \mathrm{C}\right)$, (b) daily minimum and maximum relative humidity (\%), (c) dew point (\%), (d) air level and sea level pressure (atm) according to Figure 2. The aim of this network is to train the network to achieve a balanced response between input and target data. Target is obtained as rainfall percentage of this city in $\mathrm{mm}$.

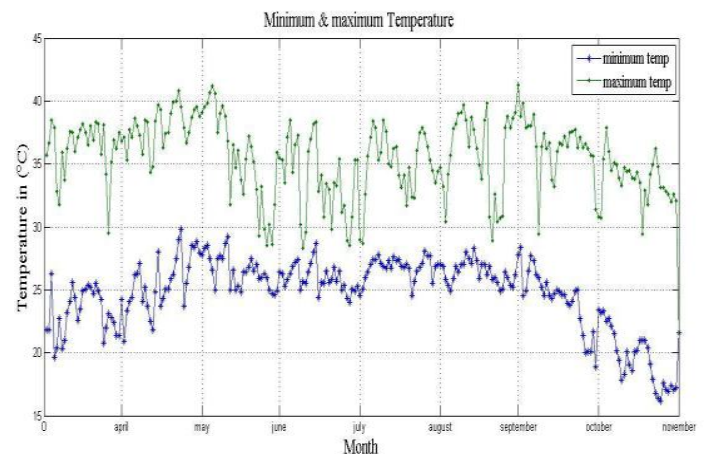

(a)

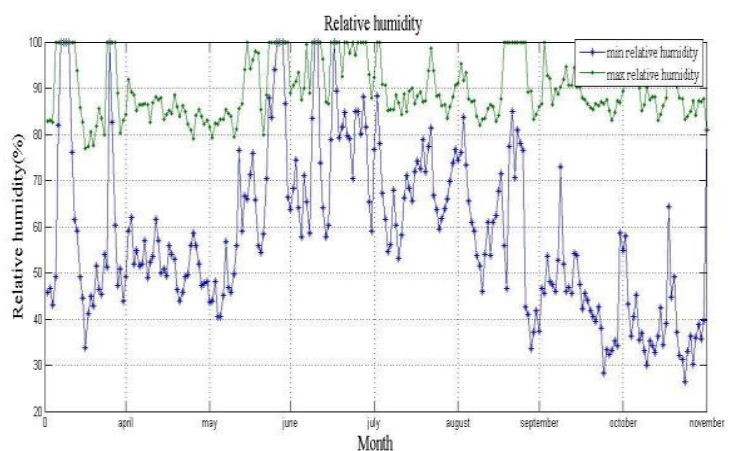

(b) 


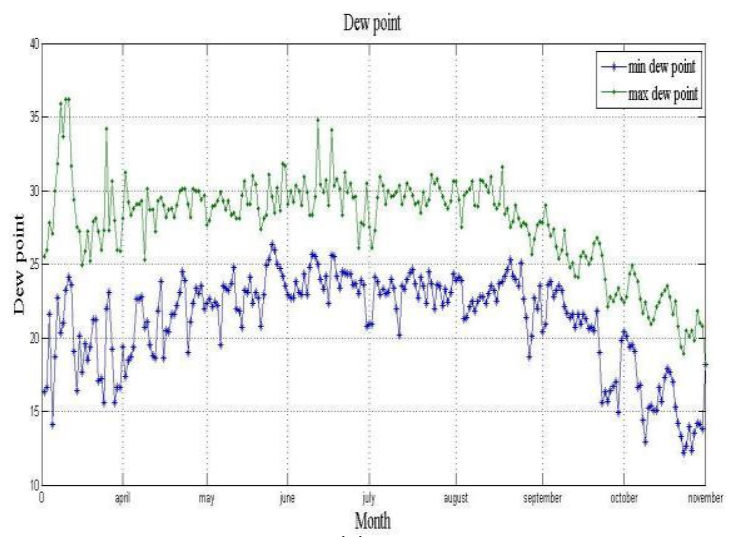

(c)

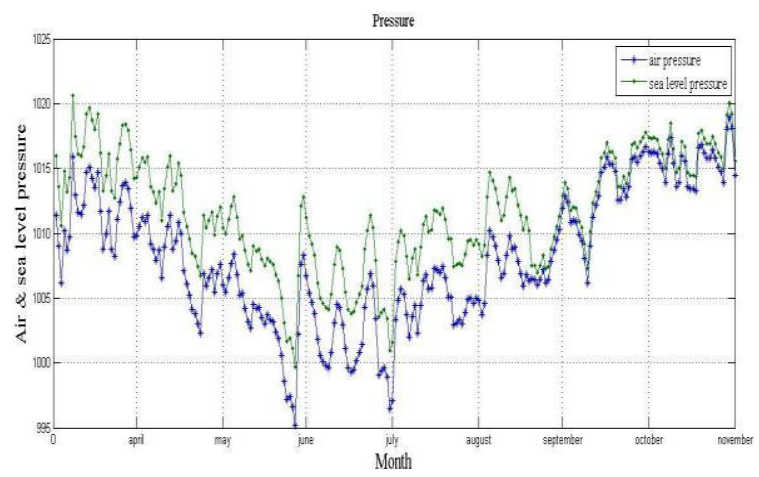

(d)

Figure 2. Collected data at 2015 (a) daily minimum and maximum temperature (b) daily minimum and maximum relative humidity (c) daily minimum and maximum dew point (d) daily air and sea level pressure.

Designing of an ANN models include the basic steps of collecting data, pre-processing data, building the network, train, and test performance of model as presented in Figure 3. Normalization of collected data is done by using premnmx function in Matlab 2013.

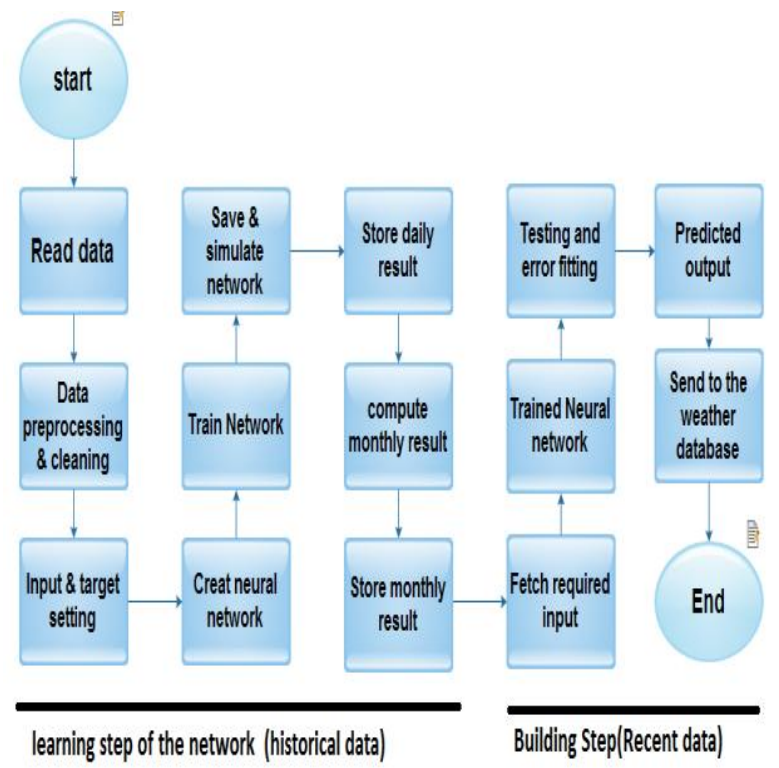

Figure 3. The proposed model using Neural Network
Normalization is included to overcome the uncertainty due to mixing criteria for the learning algorithm in Matlab platform. Neural network checks the behaviour of that non-linear data set and predicts weather condition as shown in Figure 3 for disaster possibility. A comparison between actual and predicted value is carried out through Mean Squared Error (MSE).

According to the model given in Figure 3, every weather station like satellite and GIS, local weather station and radar station data will send to central database. Then analysis will be held using ANN model to forecast disaster information for long term basis. The weights are adjusted during the training process to make the predicted outputs close to the measured outputs of the network. Total of eleven parameters of eight month's data is used as input, that is $11 * 245$ sample data and target is set as daily rainfall in $\mathrm{mm}$ that is $1 * 245$ sample data. From the data set, $70 \%$ data is used as training, $15 \%$ as validation and $15 \%$ as testing.

\section{III.RESULTS AND DISCUSSIONS}

The regression based criteria is included to validate the network performance. The regression plots are shown in Figure 4 where the performances are evaluated based on the $\mathrm{R}$ value for training, validation, test and all data sets. Validation and test performances are seen with regression coefficient, $R$ value of 0.837 and 0.713 . The dashed line in each plot represents the perfect targets where the solid line in Figure 4 represents the best fit linear regression line between outputs and targets. The accuracy of the tests increases more with the considerations of large amount of data.
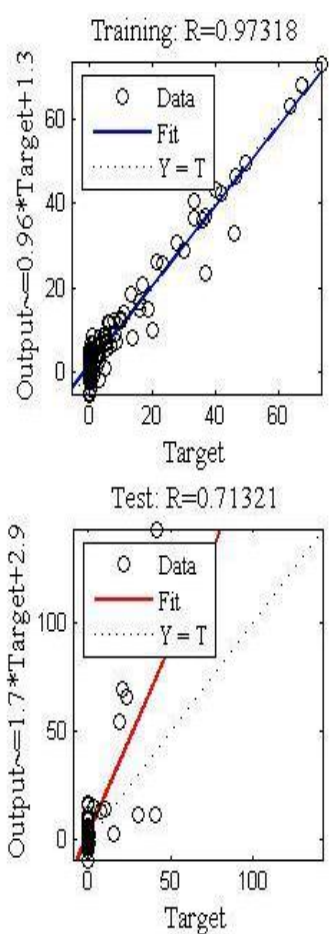

Validation: $\mathrm{R}=0.83725$

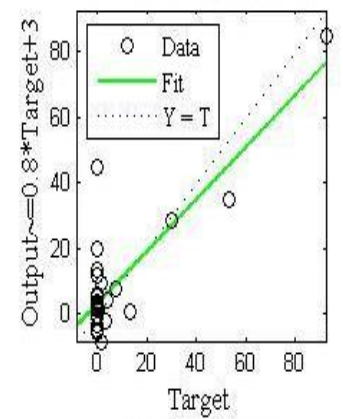

All: $\mathrm{R}=0.81832$

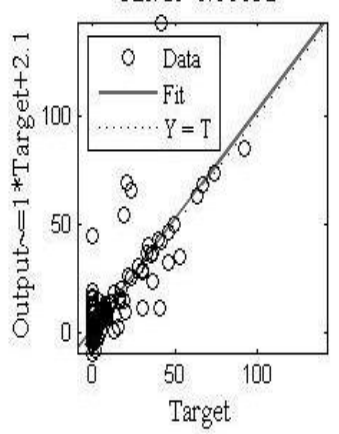

Figure 4. Regression analysis of training, test and validation data 
A plot of the training errors, validation errors and test errors can be viewed as shown in the Figure 5. In the measured network, the result is reasonable because of the small mean square error, similarity between test set error and validation set error, and absence of significant overfitting.

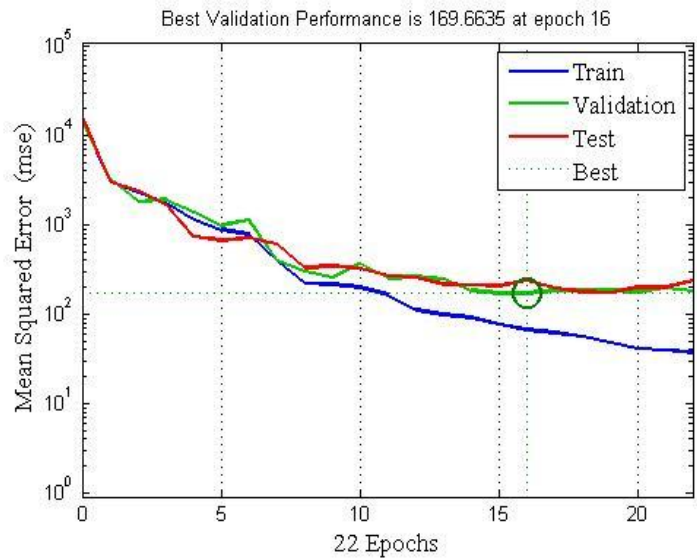

Figure 5.Performance justification with mean square error

According to Figure 5, the circle in the performance curve shows the best validation performance at epoch 16 where the best validation occurs with less mean square error. After training the network we used individual month's data for testing the result of training and forecasting the rainfall. The plotted measured and predicted rainfall of month August and September, 2015 are shown in Figure 6 to verify the model of weather prediction. According to the result seen in Figure 6, the predicted rainfall is very closer to the measured rainfall.

According to the collected data we have make some rainfall prediction. The case study includes the weather data from the month of April to November of year 2015 in Gopalganj station. So we trained the network using this data and for testing it's performance and accuracy we use the individual month's rainfall percentages of year 2015 . The outcome shows that the trained network gives similar result with respect to the measured rainfall. Using only eight month's data give us above $83 \%$ accuracy.

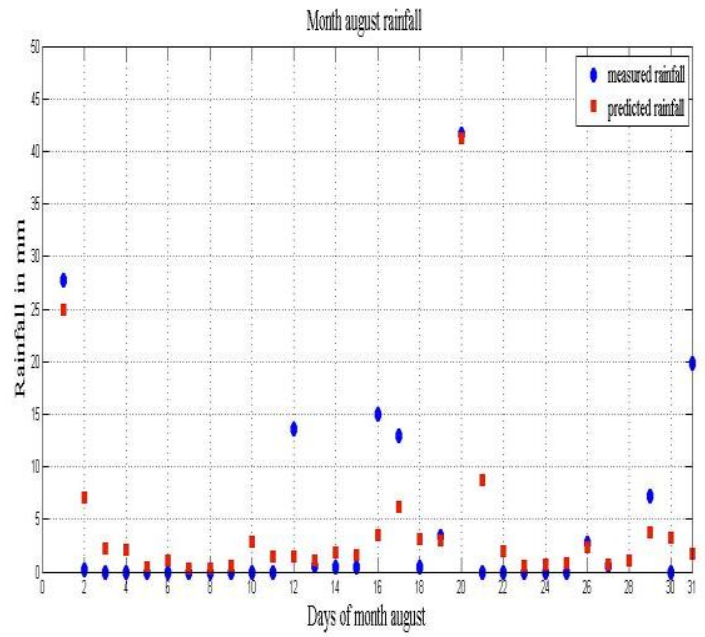

(a)

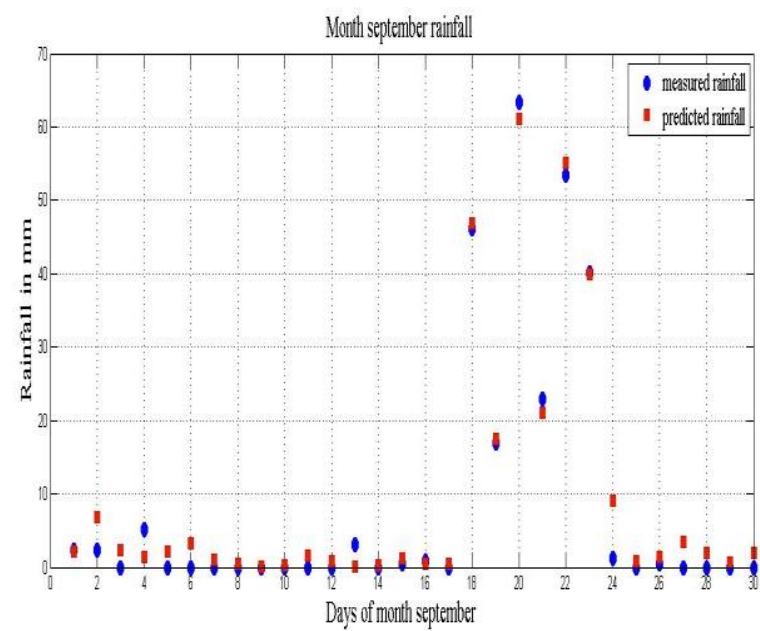

(b)

Figure 6 Comparison of measured and predicted rainfall of (a) month August (b) month September

Though huge data rate is included in the trained network of previous study, it makes the system tedious for data acquisition and processing. As an example, a lot of sensor for data acquisition ensures the costly model for disaster prediction. Since the climate change is notable for recent decade, statistics cannot handle large amount of data and differences in parameter changes the result. Therefore a database of specific and relevant weather parameters is recommended to obtain simple and cost-effective model for weather forecasting. Data normalization is included in the ANN based model to increase the reliability for seasonal disaster prediction. Figure 7 shows the predicted temperature of January, 2-16 according to the ANN model for Barisal, Bangladesh.

The reliability of the predicted temperature is also verified according to the weather information of FORECA (Android applications on Google Play) as shown in Figure 8. According to Figure 8, 24 January 2016 shows the temperature as $12^{0} \mathrm{C}$ which is very closer to the predicted temperature of $12.5^{\circ} \mathrm{C}$ for this day.

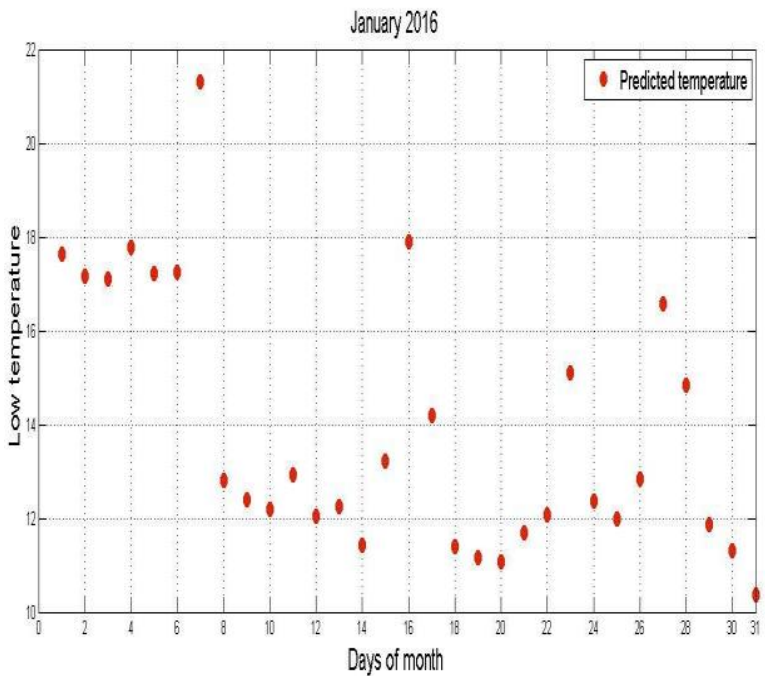

Figure 7 Predicted temperatures for January 2016 


\section{International Journal of Advanced Research in Computer and Communication Engineering}

Vol. 5, Issue 6, June 2016

In addition, the FORECA also shows the temperature of $14^{0} \mathrm{C}$ in weather information of 25 January, 2016 where the predicted temperature for that day is seen as $12^{\circ} \mathrm{C}$ in Figure 7.

Seasonal hazards cause the hammering of crop production for a wide range of agricultural area in the Bangladesh. Other areas including fisheries, forestry and environmental are also affected for the frequent disasters. Therefore reliable monthly based weather forecasting is needed to take the preparations for the forthcoming disasters.

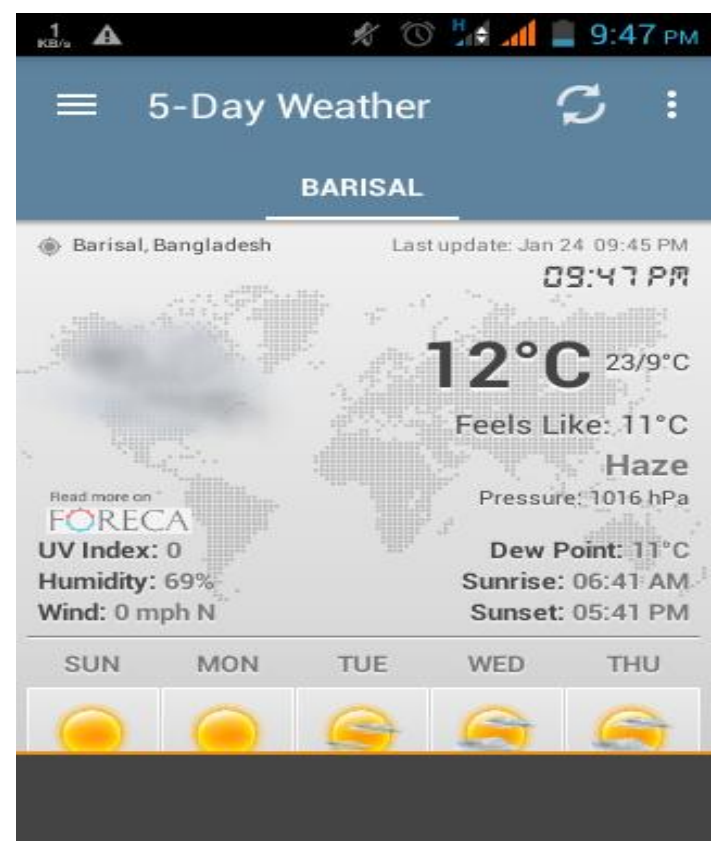

(a)

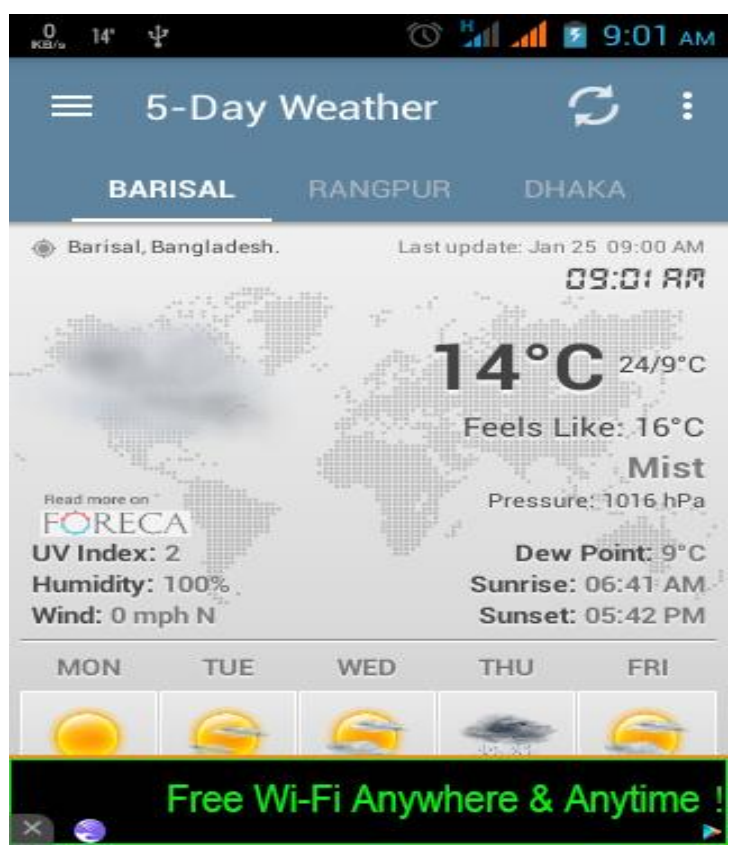

(b)

Figure 8 Daily weather information using FORECA (a) at 24 January 2016 (b) at 25 January
The research aims to obtain the simpler and reliable ANN based weather monitoring model with specific weather parameters avoiding the abundance of data acquisition and processing of meteorological data.

\section{CONCLUSION}

This paper discusses the implementation of ANN model for monthly basis reliable weather monitoring with seasonal disaster prediction. The result of given model used for monthly rainfall prediction shows that a good performance and reasonable prediction accuracy was achieved for Barisal city in Bangladesh through this model. Data normalization criteria are included to reduce the redundant data in the neural network model for seasonal disaster prediction. The mean square errors (MSE) between the actual and predicted values show that avoiding of abundant data in ANN model can perform good prediction with least error. The seasonal disaster prediction is sufficient for the preparedness of general people with the minimizing of the national economic loss.

The proposed model using ANN model has simpler and cost-effective solution with consideration of the specific weather parameters for weather forecasting.

\section{ACKNOWLEDGEMENT}

This research is conducted by the undergraduate thesis students at the Computer Science and Engineering Faculty of the Patuakhali Science \& Technology University. The authors would like to express their gratitude to all the people and associated groups that have given a lot of valuable information about the disaster in the coastal area of Bangladesh.

\section{REFERENCES}

1. Agrawal, A., Kumar, V., Pandey, A., Khan, I., 2012, "An application of time series analysis for weather forecasting," Intl. Jrnl. of Engg. Resr. and App. (IJERA), 2(2), 974-980.

2. Ahmed, S. C. (2014), "Country report of Bangladesh on effective tropical cyclone warning in Bangladesh," JMA/WMO Workshop On Effective Tropical Cyclone Warning In Southeast ASIA Tokyo, Japan 11-14 March 2014

3. Ali, M. M., Jagadeesh, P. S. V., Lin, I.-I., and Hsu, J.-Y. 2012, “A Neural Network Approach to Estimate Tropical Cyclone Heat Potential in the Indian Ocean," IEEE Geos. And Remt. Sens. Lett., 9(6).

4. Banerjee, L. 2007, "Effect of flood on agricultural wages in Bangladesh: an empirical analysis," World Dev., 35(11), 19892009.

5. Choudhury, N. Y., Paul, A. and Paul, B. K. 2004, "Impact of costal embankment on the flash flood in Bangladesh: a case study," Appl. Geogr., 24, 241-258.

6. Imran, M., Khan, M. R., Abraham, A. 2002, "Intelligent Weather Monitoring Systems Using Connectionist Models," Neural, Par. \& Sci. Comp., 10, 157-178.

7. Islam, S.M.T., and Chik, Z., 2011, "Disaster in Bangladesh and management with advanced information system," Disr. Prevn. and Manag., 20(5), 521-530.

8. Janal, P. 2009, "Operative flash floods prediction using artificial intelligence methods," Czech Hydrometeorological Institute, Kroftova 43, 61667 Brno, Czech Republic, 2009.

9. Kadu, P. P., Wagh, K. P., Chatur, P. N. 2012, “A Review on Efficient Temperature Prediction System Using Back Propagation 
Neural Network," Intl. Jrnl of Emergg. Tech. and Adv. Engg., 2(1), 52-55.

10. Karim, M. F. and Mimura, N. 2008, "Impacts of climate change and sea-level rise on cyclonic storm surge floods in Bangladesh," Glbl Envirnml Change, 1, 490-500.

11. Kumar, A. Abhay, K. Rajeev R. Sarthak, K. 2012, "A rainfall prediction model using artificial neural network," IEEE Contrl. and Syst. Grad. Resr. Collm,Shah-Alam, Malaysia, 82-87.

12. Kumar, N., Jha, G. K. 2013, "A time series ANN approach for weather forecasting," Intl. Jrnl. of Ctrl. Thry. and Com. Modeling (IJCTCM), 3(1)

13. Mahapatra, A., Nayak, D. R., Mishra, P. 2013, "A survey on rainfall prediction using artificial neural network," Intl. Jrnl. of Com. Apps (0975 - 8887), 72(16)

14. Rahman, T., Haque, T. L. 2014, "A Fuzy Neuro Based Weather Prediction System for Bangladesh," Compx. Adapt. Syst. Procd. Com. Sci., 36, 606-611.

15. Roy, C., Kovordanyi, R. 2012, "Tropical cyclone track forecasting techniques: A review," Atmos. Resr., 104-105,40-69.

16. Suliman, A., Nazri, N., Othman, M., Abdul, M. M., Mahamud, K. K., 2013, "Artificial neural network and support vector machine in flood forecasting: A review," Proceedings of the 4th International Conference on Computing and Informatics, ICOCI 2013, 28-30 August, 2013 Sarawak, Malaysia. Universiti Utara Malaysia Paper No. 030.

17. Armesh, M. and Negaresh, H. 2013, "Maximum Daily Rainfall Simulation by using Artificial Neural Network (Case Study: Saravan-Iran)" Research Journal of Environmental and Earth Sciences, 5(11), 651-659.

18. Rahman, H. and Matin, M.A. 2015, "On the Prediction of Average Monsoon Rainfall in Bangladesh with Artificial Neural Network," International Journal of Computer Applications (0975 - 8887), 127(5), 45-52. 